\title{
Detecting cognitive impairment in hospitalised patients with acute heart failure: using a sensitive tool does matter
}

Hong Kong Med J 2019;25:81-2

http://doi.org/10.12809/hkmj187772

To the Editor-Delirium is an acute deterioration of cognitive function, which frequently occurs in hospitalised elderly patients with chronic and critical diseases. ${ }^{1}$ It is associated with prolonged hospital stay and increased morbidity and mortality. Heart failure (HF) is also a major health problem in the elderly population and is one of the main reasons for hospital admission worldwide. Much evidence shows that, compared with other chronic diseases, cognitive impairment is more common in patients with HF. Delirium is a common cause of cognitive impairment in these patients, and has prognostic value for HF outcomes and deterioration of cognitive function. ${ }^{2}$ Previous research has found a positive relationship between delirium and increased risk of readmission as well as prolonged hospital stay among elderly patients with HF. ${ }^{3}$ Therefore, developing a specific screening tool for early identification of hospitalised patients at risk for cognitive impairment, and prompt management of delirium, may improve HF outcomes and quality of care.

Screening for cognitive impairment is recommended as a routine clinical assessment for patients with HF worldwide. ${ }^{4}$ However, no consensus has been achieved on the optimal screening tool for detecting cognitive impairment in hospitalised patients with HF. Currently, a number of screening tools are available for detecting delirium in the general population of older adults, ${ }^{5}$ such as the Mini-Mental State Examination (MMSE), Montreal Cognitive Assessment, and Mini-Cog. However, a more specific tool is needed for elderly patients with HF. The cognitive status of elderly patients with HF is affected by low cerebral perfusion status, which is a common condition in the pathophysiology of HF. The MMSE was originally designed for dementia screening, but most elderly patients with HF have mild cognitive impairment. In this regard, previous research showed that the MMSE is a suitable screening tool for detecting moderate to severe cognitive impairment; however, its sensitivity in detecting mild cognitive impairment is questionable. ${ }^{6}$ In addition, Montreal Cognitive Assessment can detect an unrecognised cognitive impairment in a group of stable community-dwelling patients with HF'; however, it has low specificity in ruling out mild cognitive impairment, owing to cut-offs that are inappropriate for elderly patients with HF. The applicability of the Montreal Cognitive Assessment in clinical settings, particularly in acute situations, needs to be confirmed in further research. ${ }^{7}$

Certainly, recognising cognitive impairments in elderly patients with HF is an important and challenging issue. However, developing a tailored screening tool can be a solution to improve patients' outcome. In this regard, future studies are warranted for both new instrument development and adaptation of current available tools in the context of HF.

\section{Author contributions}

The author contributed to the concept or design, acquisition of data, analysis or interpretation of data, drafting of the manuscript, and critical revision for important intellectual content. The author had full access to the data, contributed to the study, approved the final version for publication, and takes responsibility for its accuracy and integrity.

\section{Conflicts of interest}

The author has no conflicts of interest to disclose.

\section{Mohammad Ali Heidari Gorji *, PhD}

Diabetes Research Centre, Department of Medical-Surgical Nursing, Nasibeh Faculty of Nursing and Midwifery, Mazandaran University of Medical Sciences, Imam Hospital, Sari, Iran

*Corresponding author: drheidarigorij@yahoo.com

\section{References}

1. Lee AT, Chan WC, Chiu HF, et al. Physical health and lifestyle predictors for significant cognitive impairment in community-dwelling Chinese older adults in Hong Kong. Hong Kong Med J 2016;22 Suppl 6:37-9.

2. Uthamalingam S, Gurm GS, Daley M, Flynn J, Capodilupo R. Usefulness of acute delirium as a predictor of adverse outcomes in patients $>65$ years of age with acute decompensated heart failure. Am J Cardio 2011;108:402-8.

3. Ayatollahi Y, Liu X, Namazi A, et al. Early readmission risk identification for hospitalized older adults with decompensated heart failure. Res Gerontol Nurs 2018;11:190-7.

4. Cameron J, Worrall-Carter L, Page K, Riegel B, Lo SK, Stewart S. Does cognitive impairment predict poor self-care in patients with heart failure? Eur J Heart Fail 2010;12:508-15.

5. Sakaguchi T, Watanabe M, Kawasaki C, et al. A novel scoring system to predict delirium and its relationship with the clinical course in patients with acute decompensated 
heart failure. J Cardiol 2018;71:564-9.

6. Harkness K, Demers C, Heckman GA, McKelvie RS Screening for cognitive deficits using the Montreal cognitive assessment tool in outpatients $\geq 65$ years of age with heart failure. Am J Cardiol 2011;107:1203-7.
7. McLennan SN, Mathias JL, Brennan LC, Stewart S. Validity of the montreal cognitive assessment (MoCA) as a screening test for mild cognitive impairment (MCI) in a cardiovascular population. J Geriatr Psychiatry Neurol 2011;24:33-8. 